\title{
Specific survival nomograms based on SEER database for small intestine adenocarcinoma
}

\author{
Dan Wang ${ }^{1,2}$, Chenglong Li ${ }^{1}$, Yuqiang $\mathrm{Li}^{1,2}$, Wenxue Liu ${ }^{3,4}$, Lilan Zhao ${ }^{5}$, Cenap Güngör ${ }^{2}$, Fengbo Tan ${ }^{1}$, \\ Yuan Zhou ${ }^{1}$ \\ ${ }^{1}$ Department of Gastrointestinal Surgery, Xiangya Hospital, Central South University, Changsha, China; ${ }^{2}$ Department of General Visceral and \\ Thoracic Surgery, University Medical Center Hamburg-Eppendorf, Hamburg, Germany; ${ }^{3}$ Department of Rheumatology, Guangdong Provincial \\ People's Hospital, Guangdong Academy of Medical Sciences, Guangzhou, China; ${ }^{4}$ Department of Cardiology, Xiangya Hospital, Central South \\ University, Changsha, China; ${ }^{5}$ Department of Thoracic surgery, Fujian Provincial Hospital, Fuzhou, China \\ Contributions: (I) Conception and design: D Wang, F Tan, Y Zhou; (II) Administrative support: C Güngör, F Tan, Y Li; (III) Provision of study \\ materials or patients: W Liu, L Zhao; (IV) Collection and assembly of data: C Li, L Zhao; (V) Data analysis and interpretation: D Wang, F Tan; (VI) \\ Manuscript writing: All authors; (VII) Final approval of manuscript: All authors. \\ Correspondence to: Yuan Zhou. Department of Gastrointestinal Surgery, Xiangya Hospital, Central South University, 87 Xiangya Road, Changsha, \\ China. Email: doctorzhouyuan@163.com.
}

Background: Small intestine cancers, as an extremely rare tumor type, account only for $3 \%$ of all gastrointestinal tumors. Small intestine adenocarcinoma (SIA), representing approximately one-third of all small bowel cancers, has received relatively little attention, both in research efforts and clinical cognizance. Owing to anatomical proximity and rarity, small bowel adenocarcinomas are frequently grouped with colorectal adenocarcinomas. Therefore, a large SIA patient cohort is needed to develop and validate new nomogram prognostic models specific to SIA patients.

Methods: Patients diagnosed with SIA between 2004 and 2016 were extracted from the Surveillance, Epidemiology, and Final Results (SEER) database. All patients were randomly assigned to the training cohort and the validation cohort $(2: 1)$. The basic clinical information, detailed pathological staging, and treatment information of the patients were included in the analysis. Nomograms were shaped following the evaluations of the Cox regression model and verified using the decision curve analysis (DCA), time-dependent receiver operating characteristic (ROC) curves, concordance index (C-index), and calibration curves.

Results: The entire group comprised 6,947 patients with small intestine adenocarcinoma. According to the results of the multivariate Cox regression analysis, ten variables, including marital status, age, pathological grade, tumor location, $\mathrm{T}$ (tumor), $\mathrm{N}$ (nodes), $\mathrm{M}$ (metastasis) stage, surgery, chemotherapy, and regional nodes examined (RNE), were independent predictors of both of overall survival (OS) and cancer-specific survival (CSS). All significant variables were used to create the nomograms for OS and CSS. Various methods verified the reliability of the nomograms. The C-indexes of the OS and CSS nomogram were 0.756 (95\% CI, 0.748-0.764) and 0.771 (95\% CI, 0.761-0.781) in the training cohort and 0.748 (95\% CI, 0.736-0.760) and 0.767 (95\% CI, $0.752-0.781)$ in the validation cohort. The calibration curve showed good agreement between the nomogram prediction and actual survival. DCA indicated a clear net benefit of these new forecasting models.

Conclusions: This study built and verified nomograms to predict OS and CSS for rare SIA, which appear to be excellent tools to augment the clinically available evidence to facilitate the discussion between SIA patients and clinicians regarding therapeutic choice.

Keywords: Small intestine adenocarcinoma (SIA); overall survival; cancer-specific survival; nomogram; SEER database

Submitted Mar 18, 2021. Accepted for publication Jun 11, 2021.

doi: 10.21037/apm-21-600

View this article at: https://dx.doi.org/10.21037/apm-21-600 


\section{Introduction}

The small intestine accounts for more than $75 \%$ of the gastrointestinal tract and $90 \%$ of its mucosal surface. Nonetheless, small intestine cancer, as an extremely rare tumor type, accounts for only $3 \%$ of all gastrointestinal tumors (1). With an estimated 3,600 new cases per year diagnosed in Europe and 5,300 new cases per year in the USA $(2,3)$, small intestine cancers have a comparable incidence rate to testicular cancer, Hodgkin lymphoma, chronic myeloid leukemia, and anal cancer (1). The common histological types of small bowel tumors include carcinoids, adenocarcinomas, lymphomas, and sarcomas. Small intestine adenocarcinoma (SIA), representing approximately one-third of all small bowel cancers (4), receives relatively little attention, both in research efforts and clinical cognizance.

Owing to anatomical proximity and rarity, the clinical management for SIA follows that of colorectal adenocarcinomas. Despite several notable molecular similarities $(5,6)$, SIA differs from colorectal cancer (CRC) in that it involves the low bacterial load, dilute liquid contents, higher levels of lymphoid aggregates and IgA levels (7) and worse outcomes $(8,9)$. Therefore, it is necessary to better predict the prognosis of SIA patients under the existing diagnosis and treatment models.

Previous studies have suggested that factors affecting the prognosis of SIA patients include age, tumor stage, surgery, radiotherapy and chemotherapy (2). However, these variables have only been used as single indicators, which cannot accurately predict the survival of SIA patients. To overcome the limitations of a single predictor, a new nomogram prediction model was needed. Nomogram refers to visible representations of mathematical models that can combine certain features to estimate specific endpoints. The practical graphical display of the nomogram allows us to make easy and prompt predictions in clinical practice. Considering the rarity of SIA, large databases, such as the Surveillance, Epidemiology, and End Results database (SEER database), are excellent resources that can provide some necessary clinical data. SEER database has been widely used to examine the incidence and outcome patterns of various familiar cancers.

Therefore, prognostic nomograms for patients with SIA were created to assess overall survival (OS) and cancerspecific survival (CSS) based on the SEER database.

We present the following article in accordance with the TRIPOD reporting checklist (available at https://dx.doi. org/10.21037/apm-21-600).

\section{Methods}

\section{Subject selection}

A retrospective analysis involved 7,831 pathologically diagnosed SIA patients in the SEER database from 2004 to 2016. Patients with small intestine adenocarcinoma (ICD-O-3: $8140,8143,8144,8145,8210,8220,8211,8255,8260$, $8261,8262,8263,8310,8480,8481,8490)$ were the target population in this study. The exclusion criteria were as follows: (I) diagnosed at autopsy or death certificate; (II) survival months $=0$; (III) tumor size $=0$; (IV) all of the $\mathrm{T}, \mathrm{N}$, and $\mathrm{M}$ stages were blank. Exclusion process are displayed in Figure 1. The random grouping was then executed at a ratio of $2: 1$ (training group, $n=4,631$, and validation group, $n=2,316$ ).

\section{Prognostic variables}

The information involving gender, marital status, age at diagnosis, race, histological type, grade, $\mathrm{T}$ (tumor), $\mathrm{N}$ (nodes), $M$ (metastasis) stages, surgery, regional nodes examined (RNE), radiotherapy, and chemotherapy were acquired for each patient. The tumor sites of the patients were classified as duodenum, jejunum, ileum, and unknown. T stage was divided into T1-2, T3-4, and Tx. N stage and M stage were described as N0, N+, Nx, and M0, M1, Mx. Histologic type was classified as adenocarcinomas and mucinous cell carcinoma/signet ring cell carcinoma. Based on previous data and experience with lymph node dissection of colorectal cancer, the frequency of lymph node examination was divided into $0-4,4-7,8-11, \geq 12$, and unknown. All patients were inconsistently separated into two groups (training group, $\mathrm{n}=4,631$ and validation group, $\mathrm{n}=2,316$ ).

\section{Follow up}

In this study, OS and CSS were taken as endpoints. OS was defined as the time interval between the first diagnosis and death from any cause. CSS was defined as the time interval between the first diagnosis and death specific to SIA. We analyzed the 1-year, 3-year, and 5-year CSS and OS.

\section{Statistical analysis}

First, SIA patients meeting the inclusion criteria were randomly assigned at a $2: 1$ ratio to the training group $(n=4,631)$ or validation group $(n=2,316)$ using the randomization function in SPSS 26.0. In addition, SPSS 26.0 software was used for univariate and multivariate Cox 


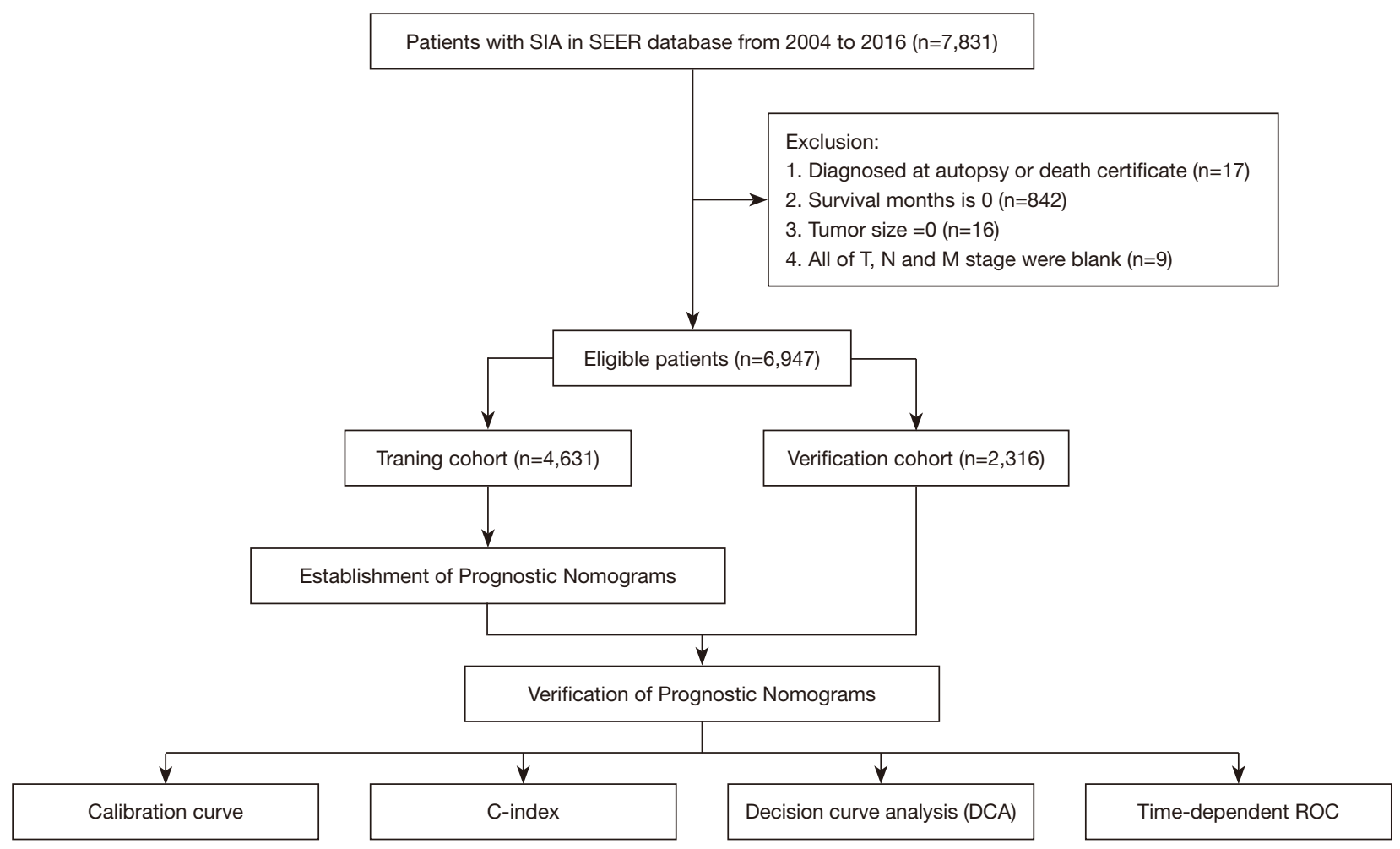

Figure 1 The workflow chart.

proportional risk regression analyses to assess and identify independent prognostic factors. The $\mathrm{P}$ value in the Cox regression model was set at 0.1 in univariate analysis. Additionally, all $\mathrm{P}$ values of less than 0.05 were considered significant. Variables were calculated using hazard ratios (HR) and corresponding 95\% confidence intervals (CI).

We utilized the $\mathrm{R}$ statistical software version 3.5 (http:// www.r-project.org) with the survival and RMS package to construct the histogram and the risk regression software package to evaluate the performance of the histogram. Various methods including decision curve analysis (DCA), time-dependent receiver operating characteristic (ROC) curves, concordance index (C-index) as well as calibration curves were used to verify the differential advantage of the histogram. The study was conducted in accordance with the Declaration of Helsinki (as revised in 2013).

\section{Results}

\section{Characteristics of patients with SIA}

The entire cohort comprised 6,947 patients with small intestine adenocarcinoma. The characteristics of the
SIA patients in this study are displayed in Table 1. The cohort comprised predominantly elderly patients $(>60$, $69.47 \%$ ) with 13 -month median survival. Overall,11.75\% of patients were diagnosed with mucinous cell carcinoma (MCC) or signet ring cell carcinoma (SRCC). Duodenum was the main site of the small intestine adenocarcinoma (57.64\%). Patients with synchronous metastases accounted for $30.39 \%$ of cases. Moreover, $37.11 \%$ of patients missed surgical resection, and only $43.70 \%$ of patients underwent chemotherapy.

\section{Establishment of prognostic nomograms}

Univariate Cox regression analyses were used for preliminary screening of prognostic factors, and multivariate Cox regression analyses were subsequently utilized to confirm the independent prognostic factors and the weight of factors affecting OS and CSS, presented as the odds ratio (OR). The preliminary prognostic factors ( $\mathrm{P}$ value $<0.1$ in the univariate analyses) were included in the multivariate Cox regression model for analysis. Ten variables, including age, marital status, tumor location, pathological grade, $\mathrm{T}$ stage, $\mathrm{N}$ stage, $M$ stage, surgery, chemotherapy, and RNE, were confirmed 
Table 1 Characteristics of patients with small intestine adenocarcinoma in the training and validation group

\begin{tabular}{|c|c|c|c|c|c|c|}
\hline Characteristics & \multicolumn{2}{|c|}{ Total $(\mathrm{n}=6,947)$} & \multicolumn{2}{|c|}{ Training group $(n=4,631)$} & \multicolumn{2}{|c|}{ Validation group $(n=2,316)$} \\
\hline \multicolumn{7}{|l|}{ Gender } \\
\hline Female & 3,183 & 45.82 & 2,105 & 45.45 & 1,078 & 46.55 \\
\hline Male & 3,764 & 54.18 & 2,526 & 54.55 & 1,238 & 53.45 \\
\hline \multicolumn{7}{|l|}{ Age (years) } \\
\hline$\leq 50$ & 849 & 12.22 & 579 & 12.50 & 270 & 11.66 \\
\hline $51-60$ & 1,272 & 18.31 & 836 & 18.05 & 436 & 18.83 \\
\hline $61-70$ & 1,815 & 26.13 & 1,216 & 26.26 & 599 & 25.86 \\
\hline$>70$ & 3,011 & 43.34 & 2,000 & 43.19 & 1,011 & 43.65 \\
\hline Unmarried/NOS & 3,095 & 44.55 & 2,078 & 44.87 & 1,017 & 43.91 \\
\hline \multicolumn{7}{|l|}{ Race } \\
\hline White & 5,211 & 75.01 & 3,466 & 74.84 & 1,745 & 75.35 \\
\hline Black & 1,238 & 17.82 & 837 & 18.07 & 401 & 17.31 \\
\hline Other/NOS & 498 & 7.17 & 328 & 7.08 & 170 & 7.34 \\
\hline \multicolumn{7}{|l|}{ Tumor location } \\
\hline Duodenum & 4,004 & 57.64 & 2,671 & 57.68 & 1,333 & 57.56 \\
\hline Jejunum & 1,044 & 15.03 & 706 & 15.25 & 338 & 14.59 \\
\hline \multicolumn{7}{|l|}{ Pathological grade } \\
\hline III-IV & 2,131 & 30.68 & 1,429 & 30.86 & 702 & 30.31 \\
\hline Unknown & 1,304 & 18.77 & 867 & 18.72 & 437 & 18.87 \\
\hline \multicolumn{7}{|l|}{ Histologic type } \\
\hline Adenocarcinomas & 6,131 & 88.25 & 4,109 & 88.73 & 2,022 & 87.31 \\
\hline MCC/SRCC & 816 & 11.75 & 522 & 11.27 & 294 & 12.69 \\
\hline \multicolumn{7}{|l|}{ T stage } \\
\hline $\mathrm{T} 1-2$ & 1,230 & 17.71 & 818 & 17.66 & 412 & 17.79 \\
\hline T3-4 & 4,546 & 65.44 & 3,044 & 65.73 & 1,502 & 64.85 \\
\hline Tx & 1,171 & 16.86 & 769 & 16.61 & 402 & 17.36 \\
\hline
\end{tabular}

Table 1 (continued) 
Table 1 (continued)

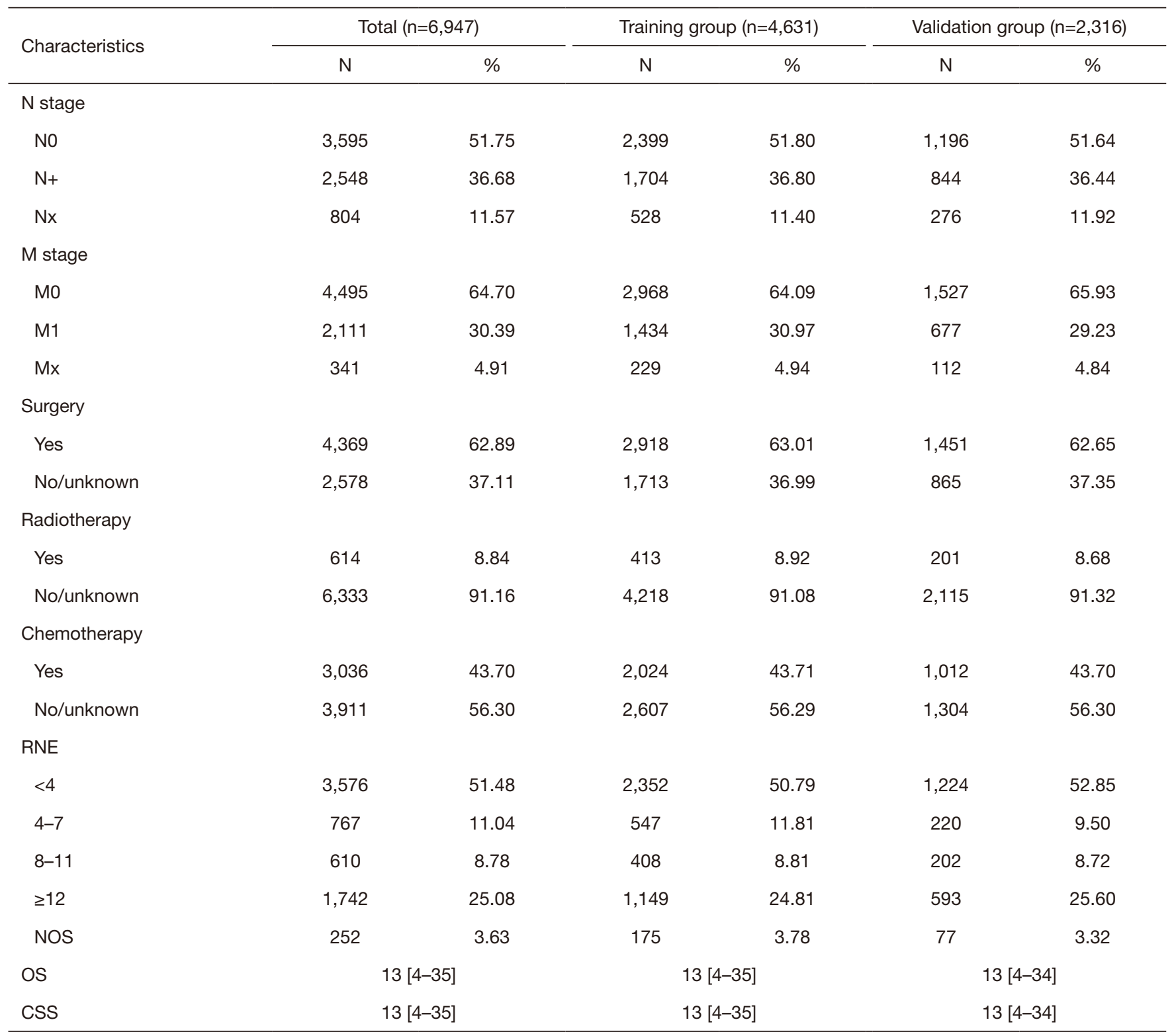

MCC, mucinous cell carcinoma; SRCC, signet ring cell carcinoma; RNE, regional nodes examined; NOS, not otherwise specified; OS, overall survival; CSS, cancer-specific survival.

as independent predictors of both OS (Table 2) and CSS (Table 3) in this study.

The nomograms predicting 1-, 3-, and 5-year OS and CSS were created using the ten variables (Figure 2). Adding up the scores related to each variable and projecting total scores to the bottom scales allowed us to easily calculate the estimated 1-, 3-, and 5-year OS and CSS probabilities.

\section{Verification of prognostic nomograms}

To identify the discriminating superiority of the nomograms, various methods involving decision curve analysis (DCA), 
Table 2 Univariable and multivariable Cox regression model analyses of OS for nomogram

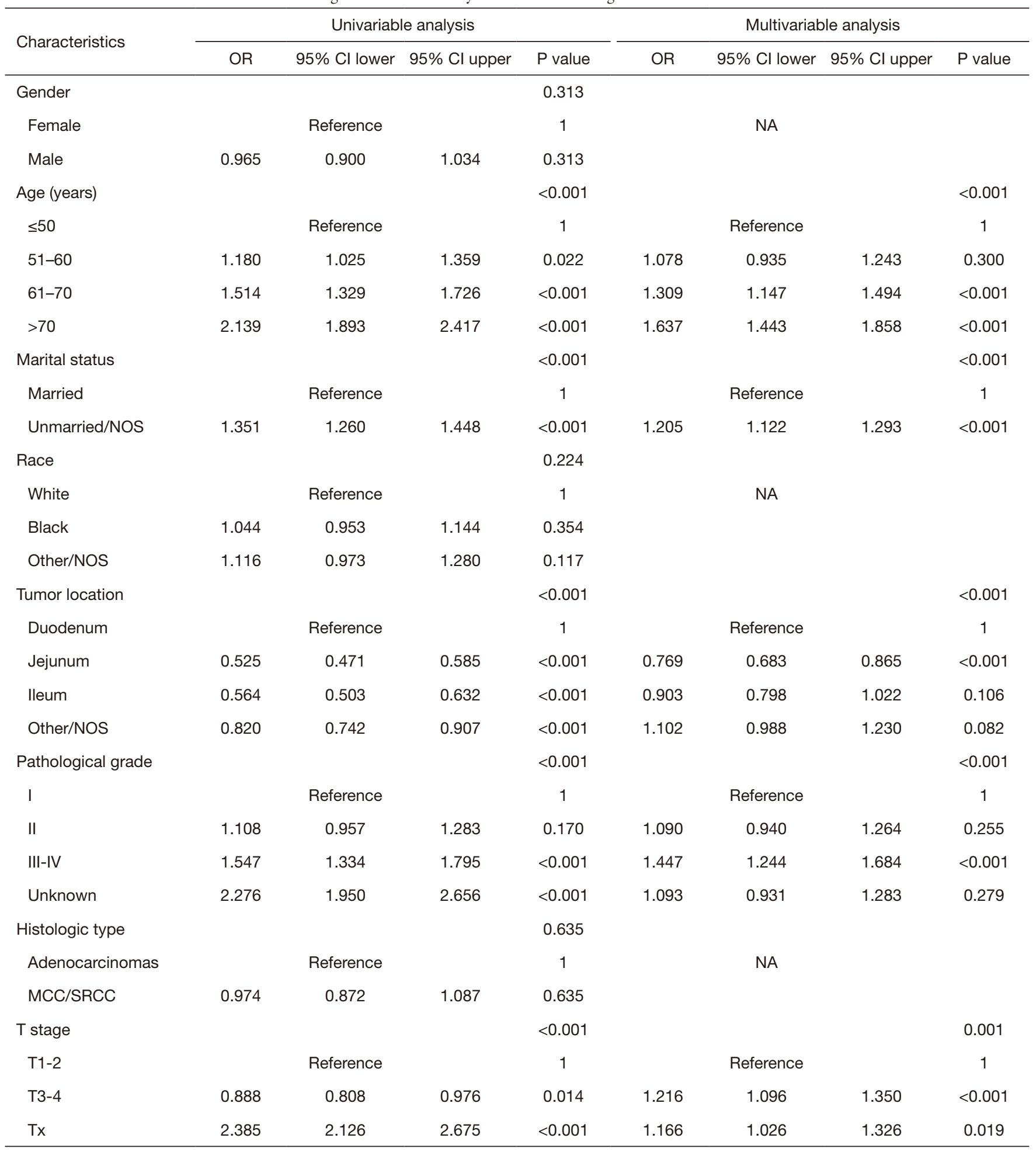

Table 2 (continued) 
Table 2 (continued)

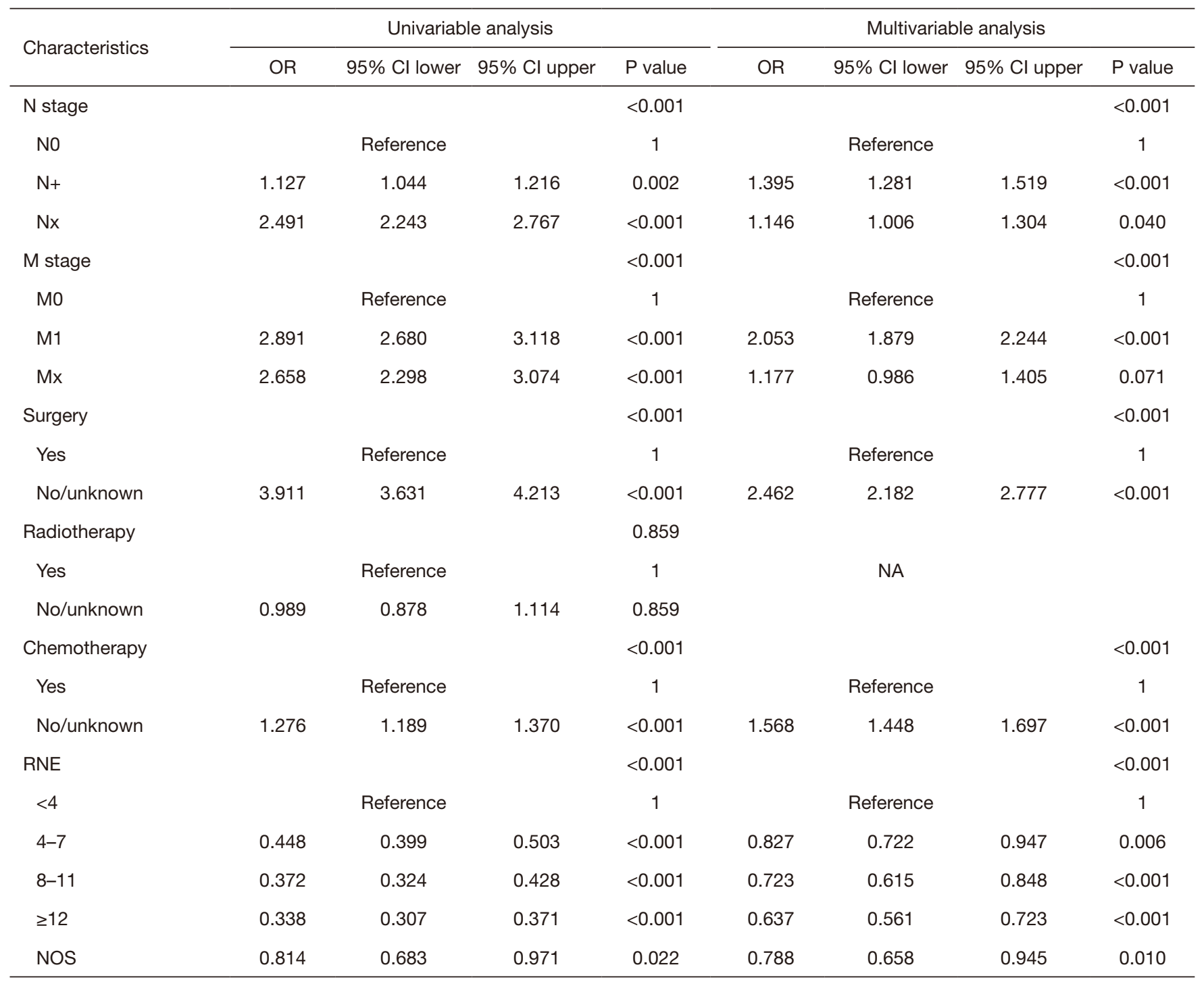

MCC, mucinous cell carcinoma; SRCC, signet ring cell carcinoma; RNE, regional nodes examined; NOS, not otherwise specified; NA, unavailable.

time-dependent receiver operating characteristic (ROC) curves, concordance index (C-index), as well as calibration curves were used in this study. The $\mathrm{C}$-indexes of the $\mathrm{OS}$ nomogram were 0.756 (95\% CI, $0.748-0.764)$ and 0.748 (95\% CI, 0.736-0.760) in the training and verification group, respectively, which were higher compared to those of the AJCC stage for OS (0.613 (95\% CI, 0.600-0.625) in the training cohort and 0.626 (95\% CI, 0.609-0.643) in the verification cohort). The differences between the nomogram and AJCC stage in the prediction of CSS were similar. The C-indexes of a nomogram predicting CSS were 0.771 (95\% CI, 0.761-0.781) in the training and 0.767 (95\% CI, 0.752-0.781) in the verification cohort. Additionally, the AJCC stage illustrated inferior value of c-index $(0.659$ (95\% CI, 0.643-0.675) in the training and 0.670 (95\% CI, $0.648-0.692)$ in the verification cohort) (Table 4). 
Table 3 Univariable and multivariable Cox regression model analyses of CSS for nomogram

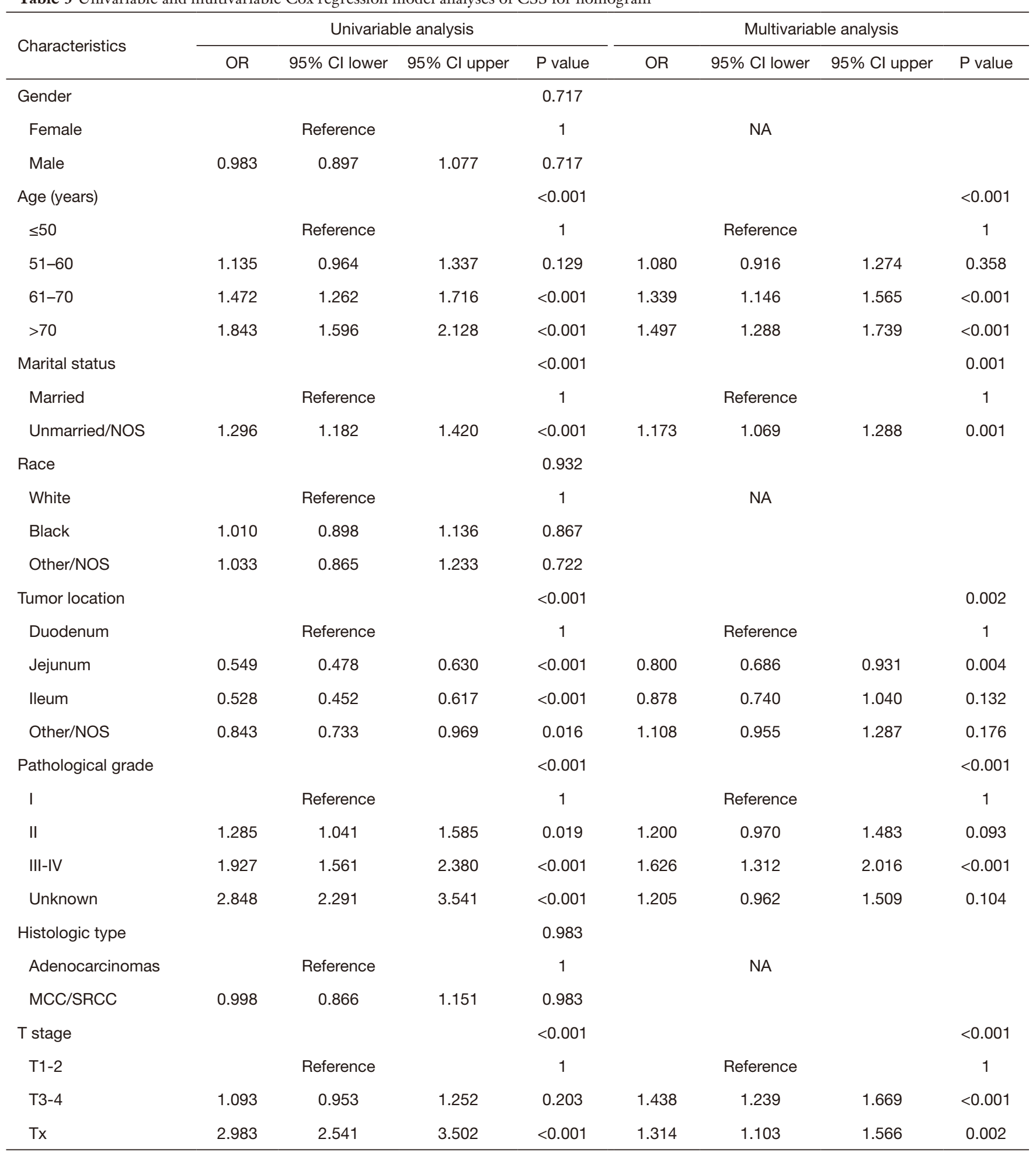

Table 3 (continued) 
Table 3 (continued)

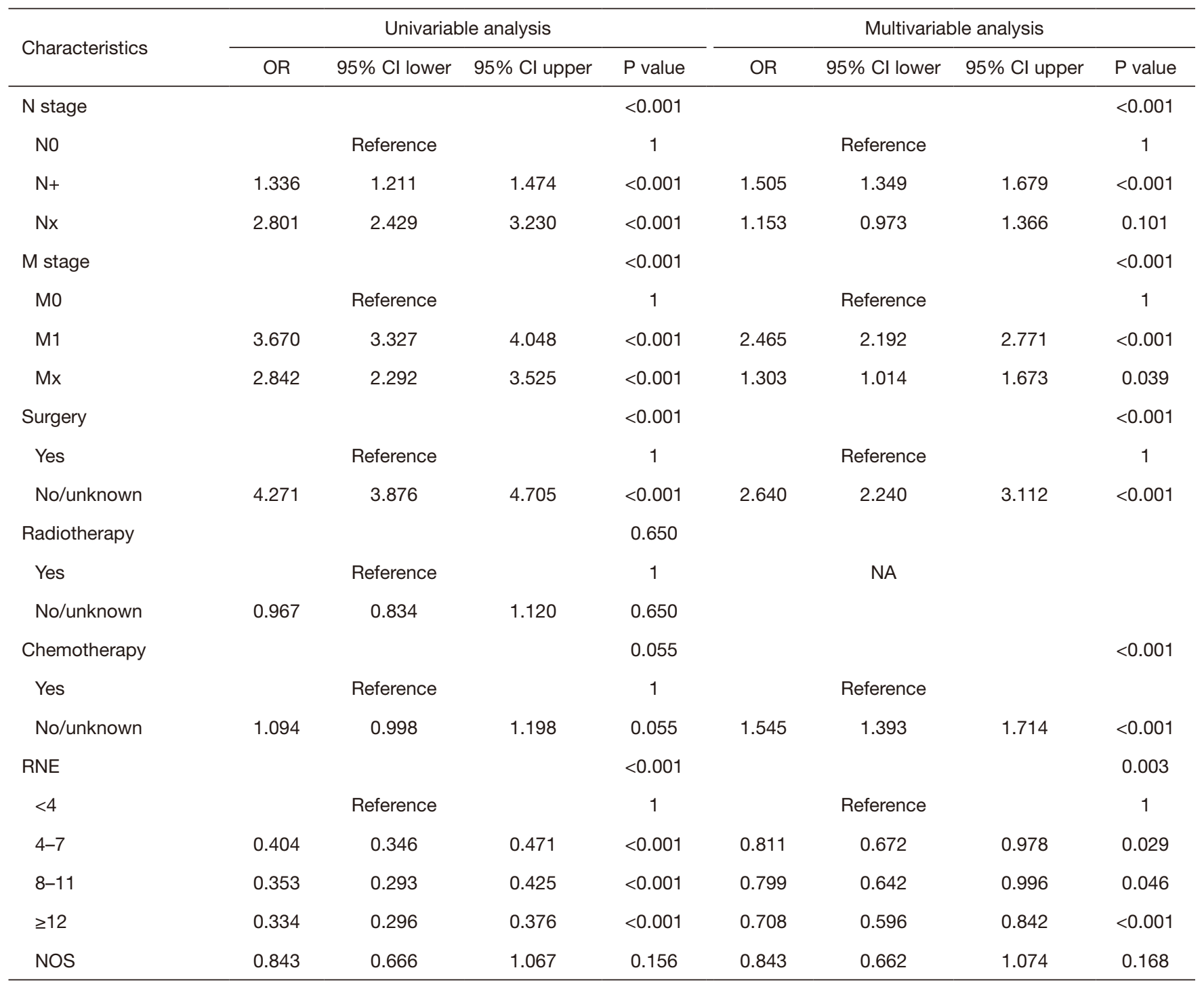

MCC, mucinous cell carcinoma; SRCC, signet ring cell carcinoma; RNE, regional nodes examined; NOS, not otherwise specified; NA, unavailable.

Time-dependent ROC at 1-, 3-, and 5-years were conducted to confirm higher sensitivity and specificity of the nomograms in predicting the prognosis of SIA patients compared to the AJCC stage. The 1-, 3-, and 5-year AUC values of the nomogram were $83.38 \%, 83.82 \%$ and $83.58 \%$ for OS compared to63.18\%, 67.85\%, and $69.13 \%$ for AJCC stage, respectively, in the training group (Figure $3 A, B, C$ ). The AUC values of the nomogram were also superior to
AJCC stage (1-year OS: $82.84 \%$ vs. $69.40 \%$; 3-year OS: $81.87 \%$ vs. $69.87 \%$; 5 -year OS: $81.33 \%$ vs. $70.62 \%$ ) in verification group (Figure $3 D, E, F$ ). In addition, the nomogram performed better for CSS compared to the AJCC stage in both of training (1-year CSS: $84.50 \%$ vs. 67.81\%; 3-year CSS: $85.59 \%$ vs. $73.13 \%$; 5 -year CSS: $85.87 \%$ vs. $75.43 \%$ ) (Figure $3 G, H, I)$ and verification cohorts (1-year CSS: $85.30 \%$ vs. $68.97 \%$; 3-year CSS: $83.10 \%$ vs. 
A

Points

Age

Marital status

Tumor location

Grade

T stage

$\mathrm{N}$ stage

$M$ stage

Surgery

Chemotherapy

RNE

Total Points

1-year OS

3-year OS

5-year OS

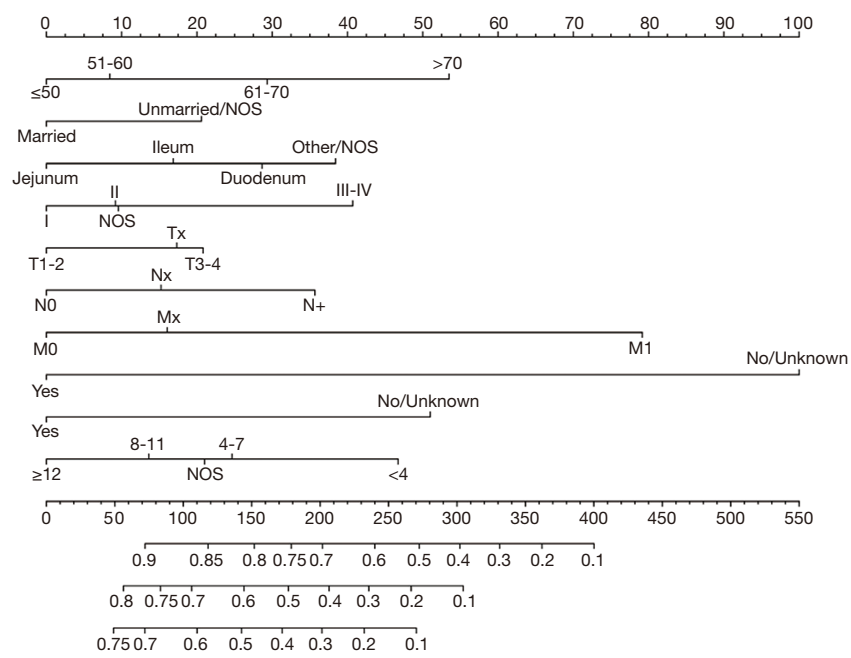

\section{B}

Points

Age

Marital status

Tumor location

Grade

T stage

$\mathrm{N}$ stage

$M$ stage

Surgery

Chemotherapy

RNE

Total Points

1-year CSS

3-year CSS

5-year CSS

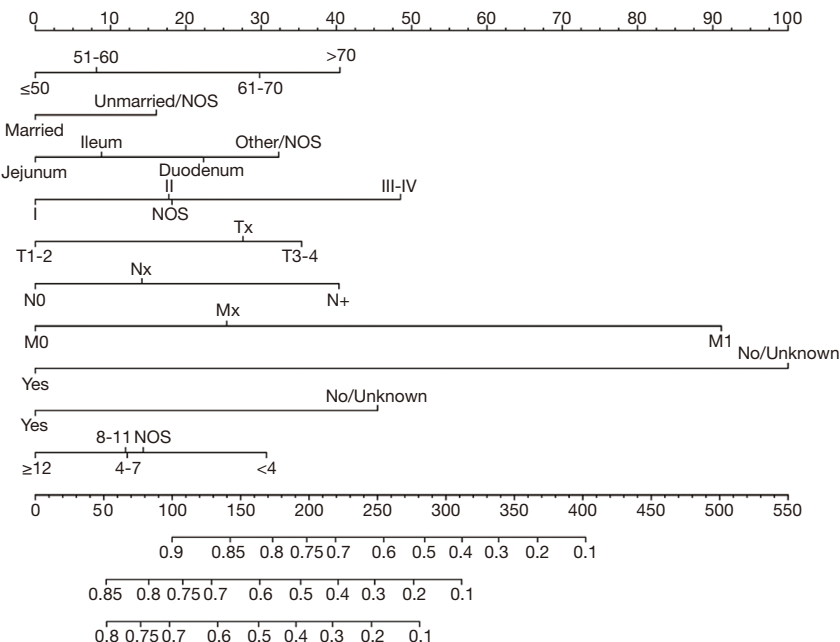

Figure 2 The nomograms for SIA patients. (A) Predicting OS. (B) Predicting CSS. SIA, small intestine adenocarcinoma; OS, overall survival; CSS, cancer-specific survival.

Table 4 The C-indices for predicting overall survival and cancer-specific survival

\begin{tabular}{|c|c|c|c|c|}
\hline Groups & \multicolumn{2}{|c|}{ OS } & \multicolumn{2}{|c|}{ cSS } \\
\hline Training group-Nomogram & 0.756 & $0.748-0.764$ & 0.771 & $0.761-0.781$ \\
\hline Training group-AJCC stage & 0.613 & $0.600-0.625$ & 0.659 & $0.643-0.675$ \\
\hline Validation group-Nomogram & 0.748 & $0.736-0.760$ & 0.767 & $0.752-0.781$ \\
\hline
\end{tabular}

OS, overall survival; CSS, cancer-specific survival; C-index, index of concordance; Cl, confidence interval. 

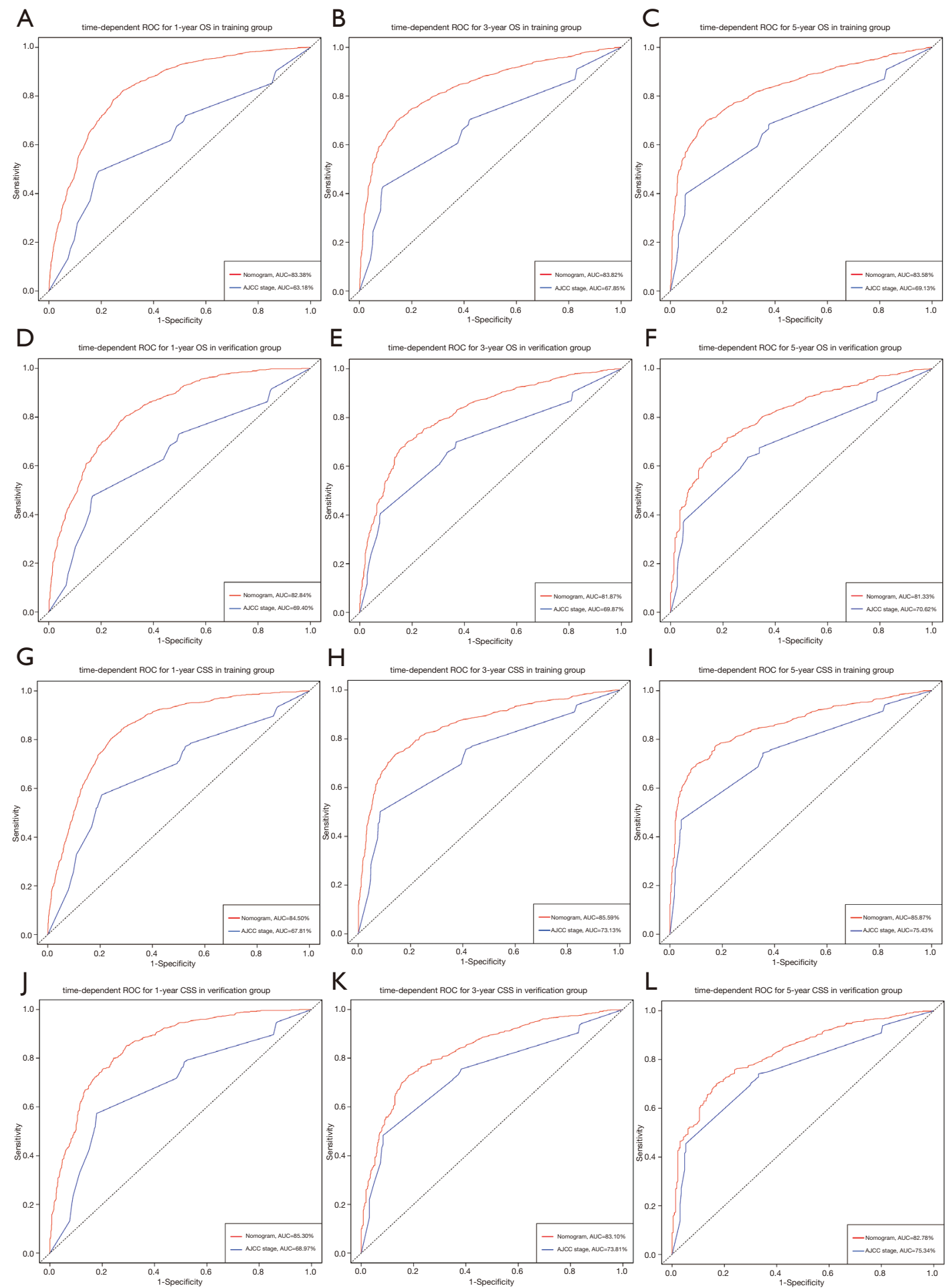

Figure 3 AUC values of ROCs of the nomograms and AJCC stage. (A,B,C) (training group): 1-year OS (83.38\% vs. 63.18\%); 3-year OS (83.82\% vs. 67.85\%); 5 -year OS (83.58\% vs. 69.13\%). (D,E,F) (verification group): 1-year OS (82.84\% vs. 69.40\%); 3-year OS (81.87\% vs. 69.87\%); 5-year OS (81.33\% vs. 70.62\%). (G,H,I) (training group): 1-year CSS (84.50\% vs. 67.81\%); 3-year CSS (85.59\% vs. $73.13 \%)$; 5 -year CSS (85.87\% vs. 75.43\%). (J,K,L) (verification group): 1-year CSS (85.30\% vs. 68.97\%); 3-year CSS (83.10\% vs. $73.81 \%)$; 5 -year CSS (82.78\% vs. $75.34 \%)$. OS, overall survival; CSS, cancer-specific survival. 

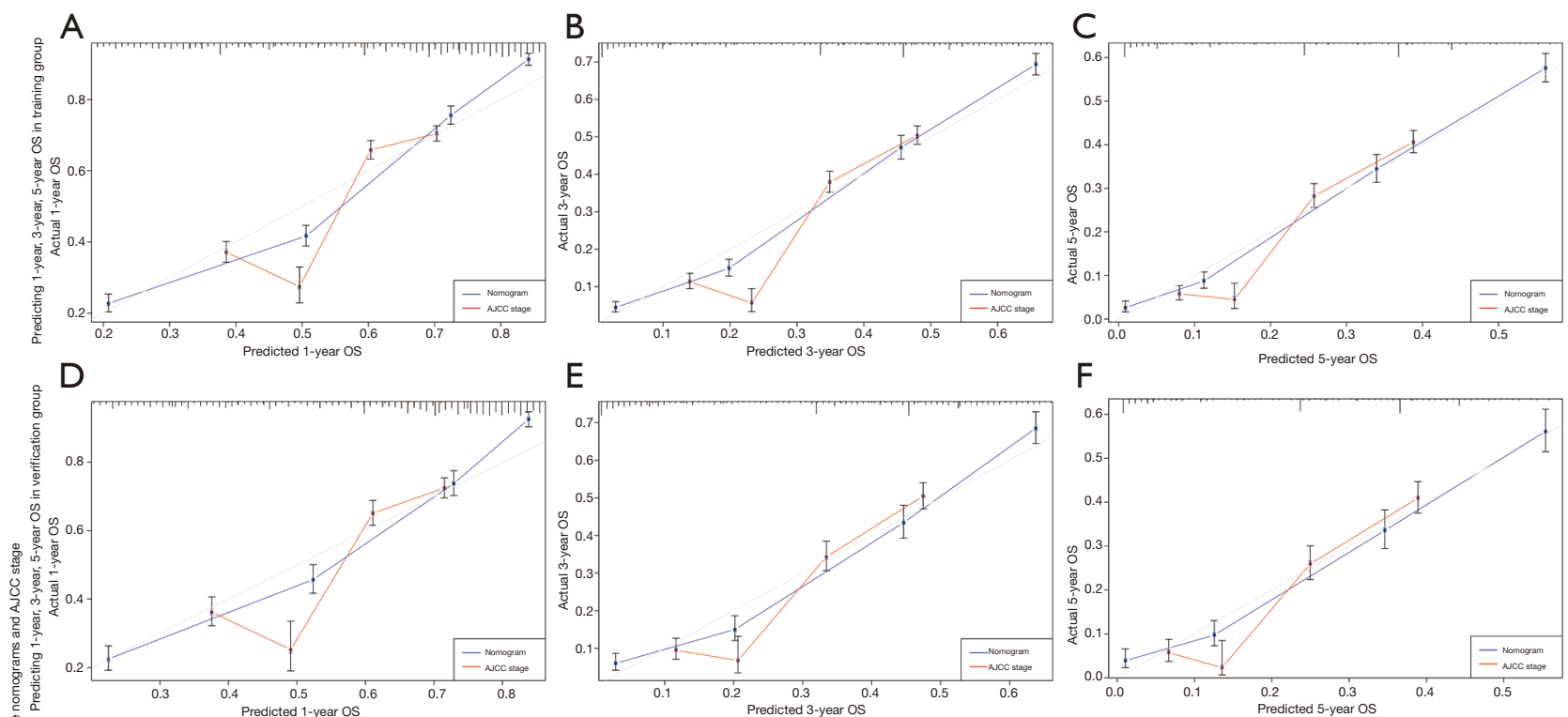

$\mathrm{E}$

$\mathrm{F}$
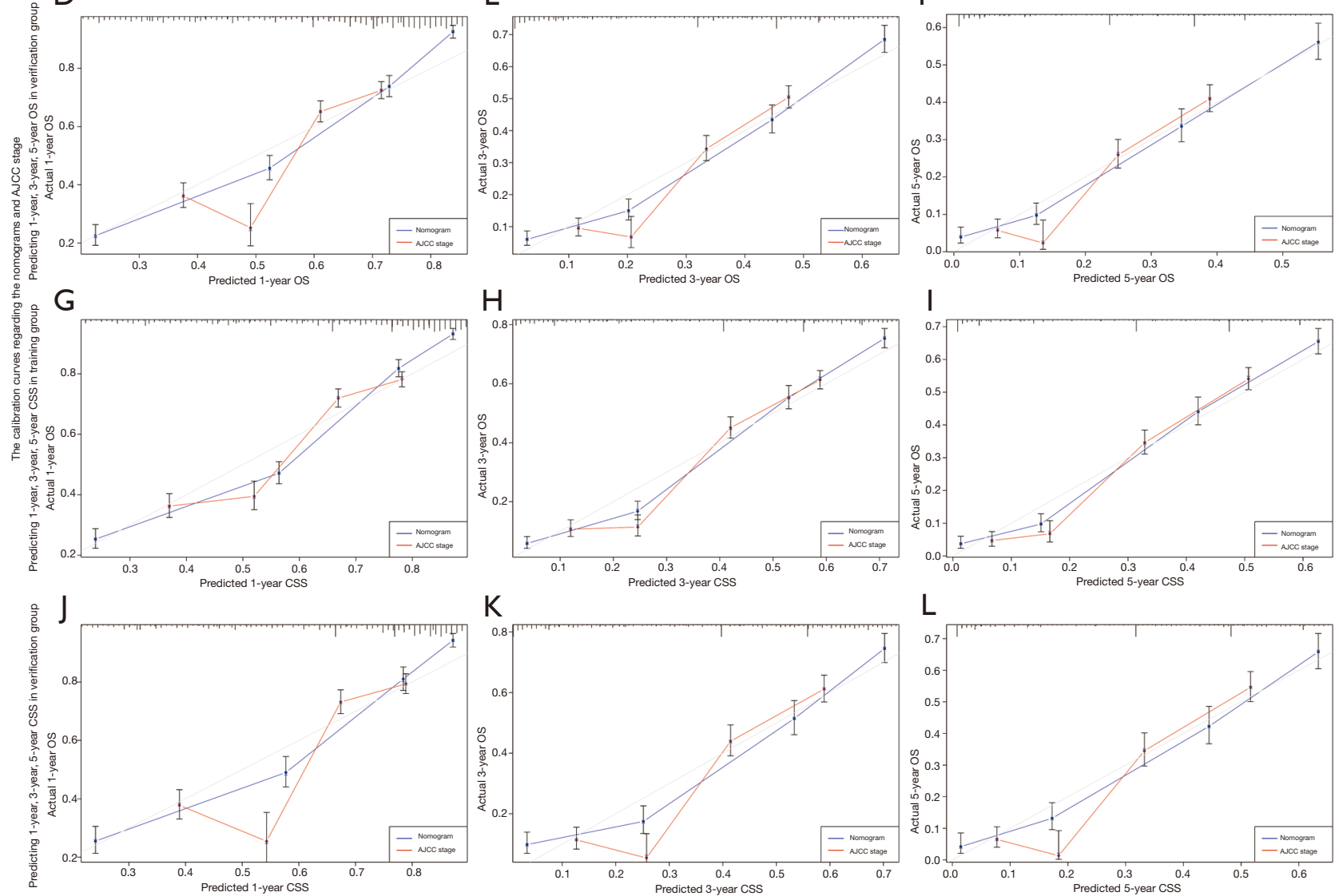

Figure 4 The calibration curves regarding the nomograms (blue lines) and AJCC stage (red lines). (A,B,C) (training group): predicting 1-year, 3-year, 5-year OS. (D,E,F) (verification group): predicting 1-year, 3-year, 5-year OS. (G,H,I) (training group): predicting 1-year, 3-year, 5-year CSS. (J,K,L) (verification group): predicting 1-year, 3-year, 5-year CSS. OS, overall survival; CSS, cancer-specific survival.

73.81\%; 5-year CSS: $82.78 \%$ vs. $75.34 \%$ ) (Figure 37,K,L).

In addition, nomograms hold the minor deviations from the reference line comparing with the AJCC stage in calibration curves for both of OS (Figure $4 A, B, C, D, E, F$ ) and CSS (Figure 4G,H,I,F,K,L), which demonstrating a high degree of reliability. DCA curves for the novel nomograms and AJCC stage are presented in Figure $5 A, B, C, D, E, F$ for OS and Figure 5G,H,I,F,K,L for CSS. Compared to the AJCC stage, the DCA of the nomograms showed superior net benefits, indicating that the nomograms in this study have a better clinical application than the AJCC stage.

\section{Risk stratification}

The prognostic scores of all independent variables were assigned based on the established nomogram, and the optimal cut-off values were calculated using X-tile based on the total scores. According to the cut-off values of the 
A

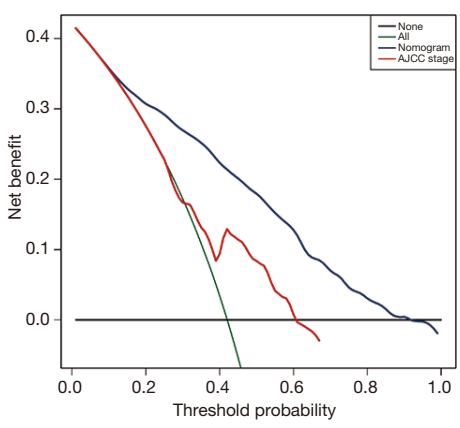

$\mathrm{D}$
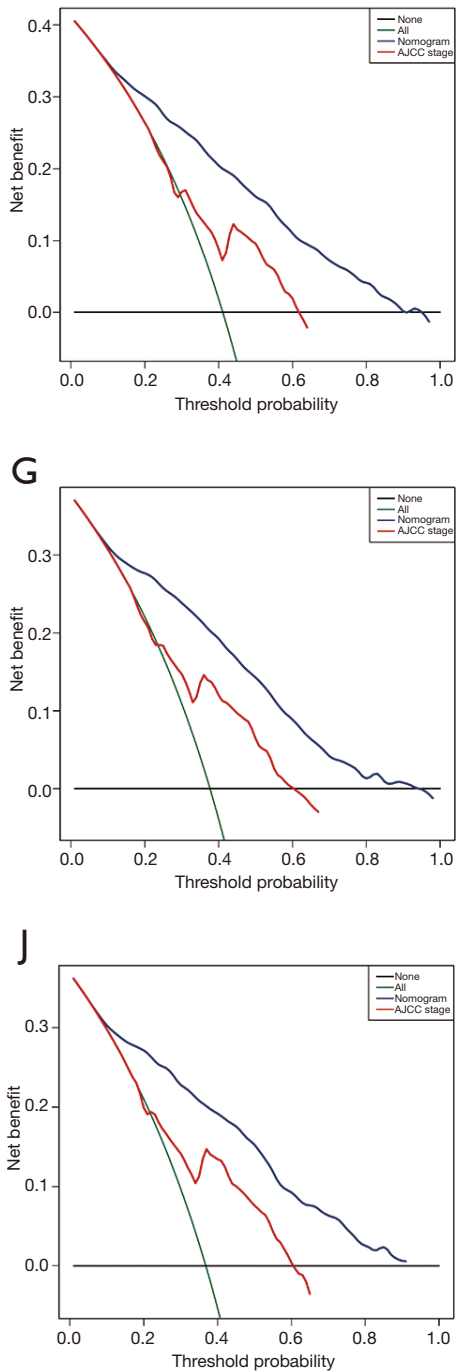

B

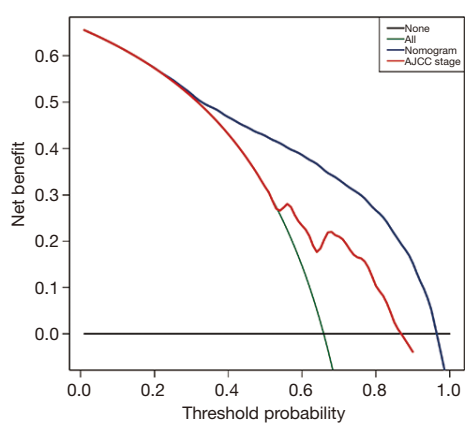

E
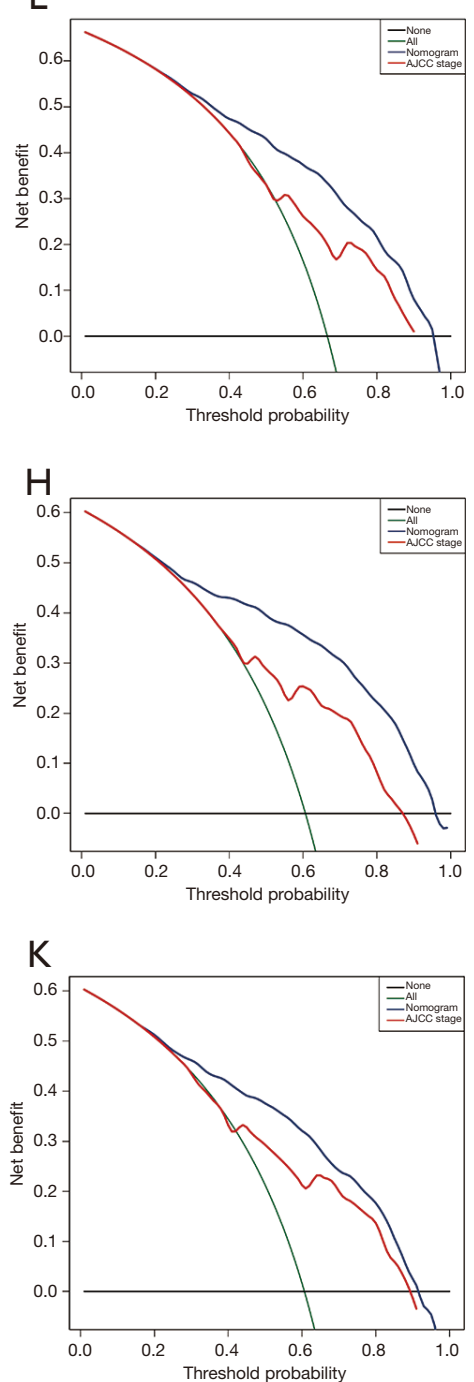

C

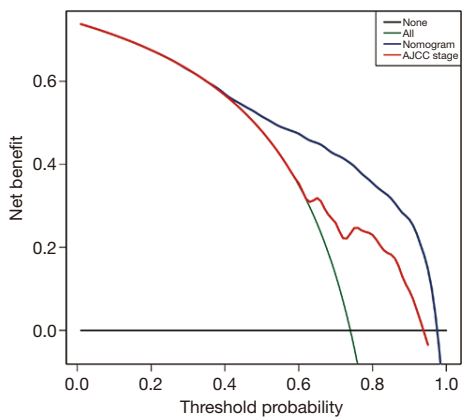

$\mathrm{F}$
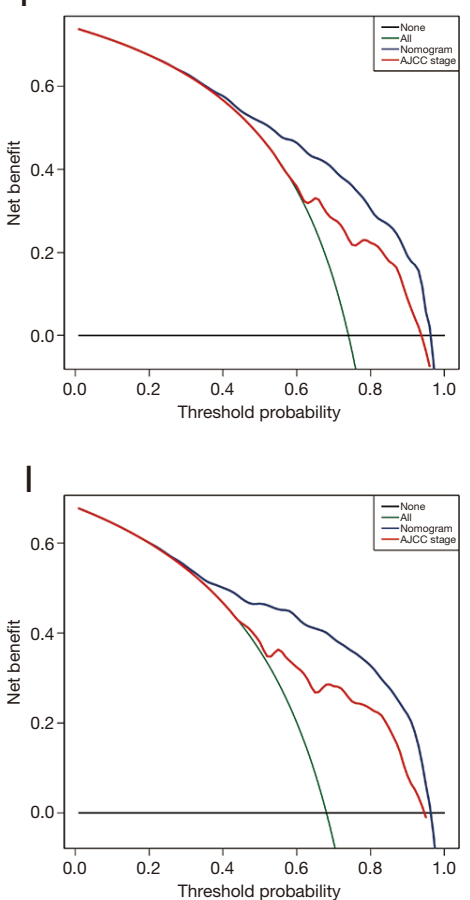

L

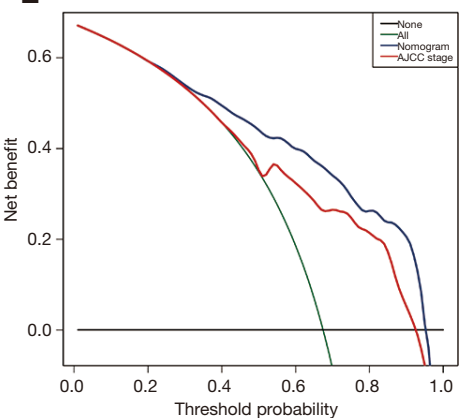

Figure 5 Decision curve analysis regarding the nomograms and AJCC stage. (A,B,C) (training group): for the 1-year, 3-year, 5-year OS. (D,E,F) (verification group): for the 1-year, 3-year, 5-year OS. (G,H,I) (training group): for the 1-year, 3-year, 5-year CSS. (J,K,L) (verification group): for the 1-year, 3-year, 5-year CSS. OS, overall survival; CSS, cancer-specific survival. 

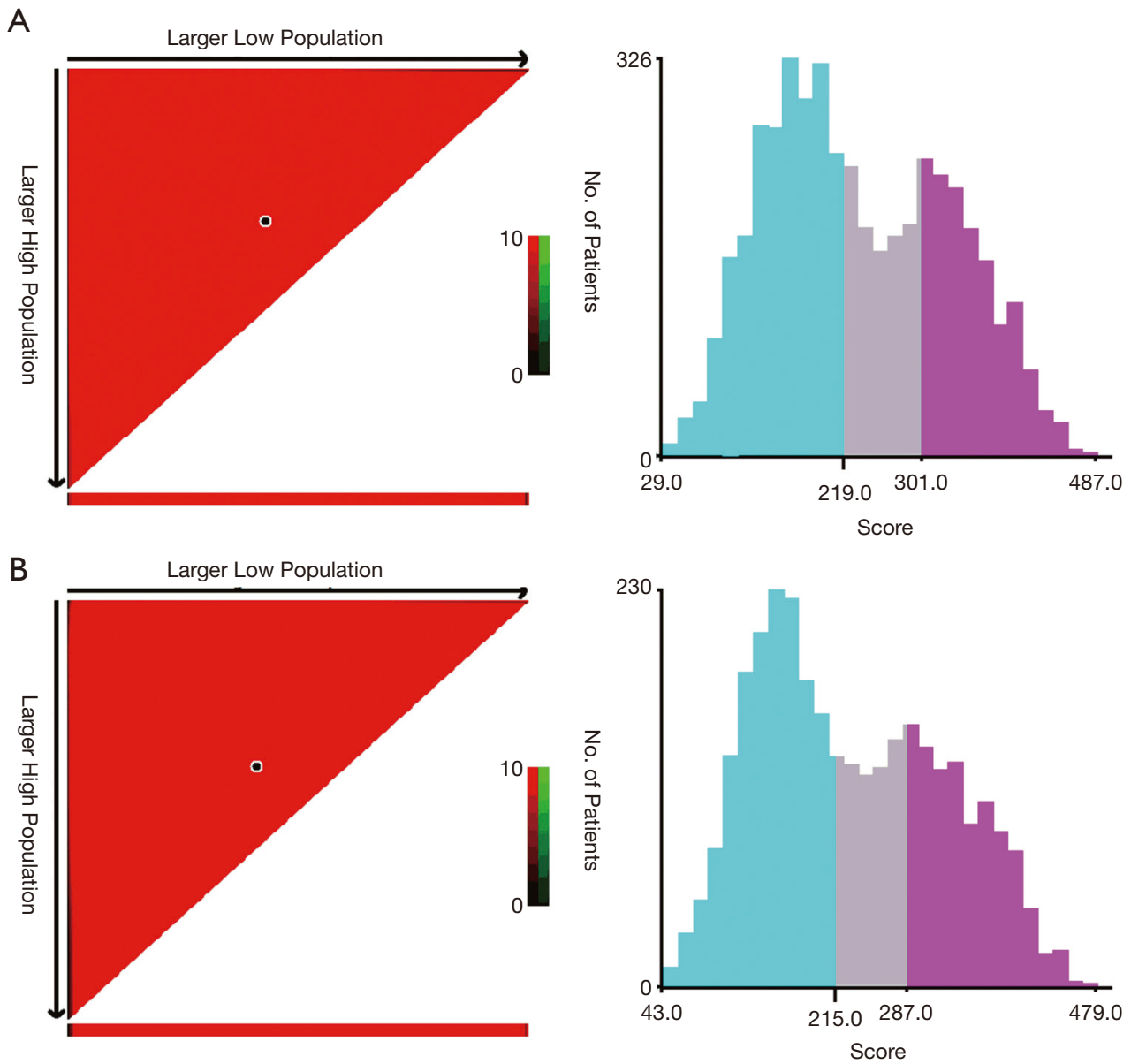

Figure 6 The cut-off values were calculated using X-tile based on the total scores. (A) The cut-off values were 219 and 301 for OS. (B) The cut-off values were 215 and 287 for CSS. OS, overall survival; CSS, cancer-specific survival.

nomogram for OS, patients with SIA were divided into low-risk (score $\leq 219)$, moderate-risk $(219<$ score $\leq 301)$, and high-risk (score $>301$ ) (Figure $6 A$ ). In addition, patients with SIA were classified as low-risk (score $\leq 215$ ), moderaterisk $(215<$ score $\leq 287)$, and high-risk (score $>287)$ for CSS (Figure 6B).

The Kaplan-Meier survival curves were subsequently delineated, as shown in Figure 7. The low-risk group had the highest 5 -year OS rate $(46.95 \%$ in training cohort and $44.61 \%$ in verification cohort), followed by the moderaterisk group $(10.07 \%$ in training cohort and $10.97 \%$ in verification cohort) and high-risk group (2.43\% in training cohort and $3.81 \%$ in verification cohort) (Figure $7 A$ and $B$ ). Similarly, the high-risk group in the training and verification cohorts had the lowest 5 -year CSS rates of $3.07 \%$ and $4.03 \%$, respectively, followed by the moderaterisk group $(12.87 \%$ in the training cohort and $17.39 \%$ in the verification cohort) and low-risk group $(55.96 \%$ in the training cohort and $53.78 \%$ in the verification cohort) (Figure $7 C$ and $D$ ). A statistically significant difference in survival outcomes was observed between the three groups $(\mathrm{P}<0.001)$.

\section{Discussion}

To our knowledge, this is the first large-database study specifically designed to describe the prognostic factors in SIA patients. This study developed and effectively validated prognostic OS and CSS nomograms for patients with SIA that could be better incorporated into clinical practice to guide surveillance and management strategies based on tumor and demographic variables.

SIA is usually diagnosed at an advanced disease stage due to the lack of specific symptoms and effective diagnostic 
A

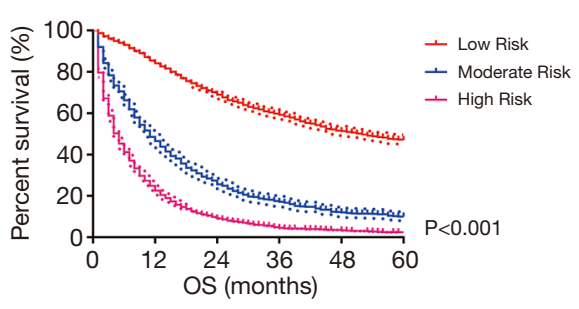

C

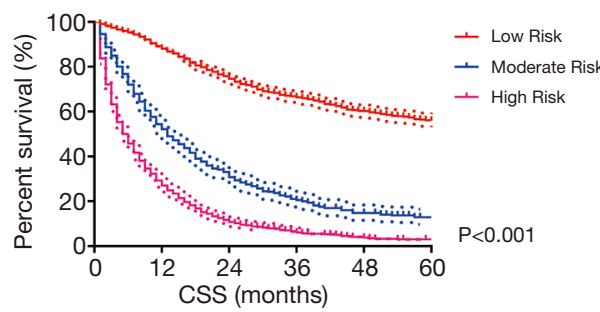

B
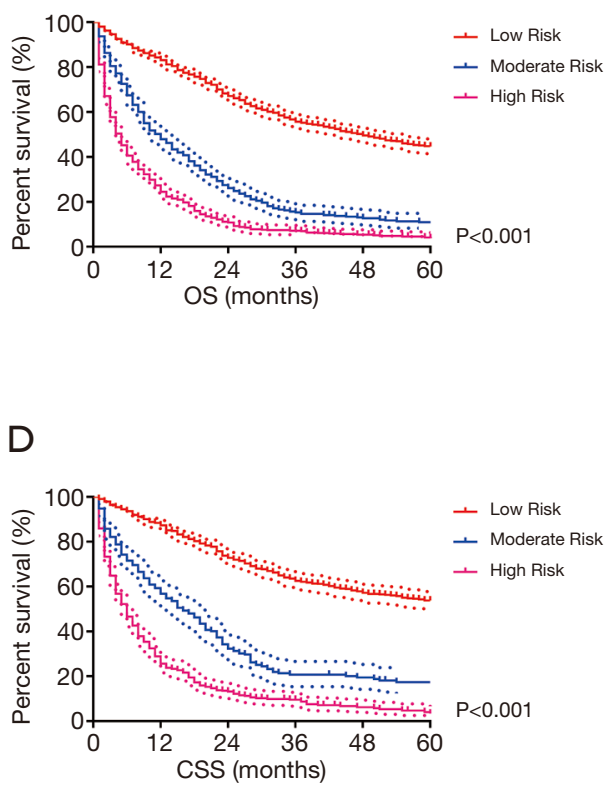

Figure 7 Survival analysis in the subgroup according to the risk stratification of the total score. (A,B) Low-risk group had the highest 5 -year OS rate $(46.95 \%$ in training cohort and $44.61 \%$ in verification cohort), followed by the moderate-risk group (10.07\% in training cohort and $10.97 \%$ in verification cohort) and high-risk group (2.43\% in training cohort and $3.81 \%$ in verification cohort). (C,D) High-risk group had the lowest 5 -year CSS rate of $3.07 \%$ and $4.03 \%$ in the training and verification cohorts respectively, followed by the moderate-risk group ( $12.87 \%$ in training cohort and $17.39 \%$ in verification cohort) and low-risk group $(55.96 \%$ in training cohort and $53.78 \%$ in verification cohort).

tools. The routine gastroduodenoscopy can assess only tumors in the proximal location of the small intestine, which explains the previous findings reporting that the rate of metastatic disease at diagnosis reached $32-33 \%$ in patients with SIA $(10,11)$, similar to this study $(30.39 \%)$. Moreover, $37.11 \%$ of SIA patients missed the surgical resection at the time of diagnosis. It is, therefore, necessary to explore tumor markers and diagnostic methods for SIA with adequate sensitivity and specificity.

The study demonstrated that tumor location was associated with survival rates. Similarly, Howe et al. (12) demonstrated worse cancer-specific survival in patients with duodenum compared to those with jejunal or ileal cancers. Nicholl et al. (13) revealed that patients with ileal tumors had a better OS compared to those with jejunal cancer by analyzing 1,444 patients with SIA. The nomograms were consistent with the results of these previous studies. Most importantly, patients with unclear tumor location suffered the greatest risk of survival among all SIA.

Based on the American Joint Committee on Cancer (AJCC) staging system, the tumor stage is the single most important prognostic factor in small bowel adenocarcinomas. The nomograms manifested clear advantages over the AJCC stage. First, the time-dependent ROC indicated that the nomograms had higher sensitivity and specificity. Second, minor deviations from the reference line demonstrated a high degree of reliability of the nomograms. Furthermore, DCA curves showed the nomograms facilitated better clinical decisions. The nomograms also considered the weight of the $\mathrm{T}, \mathrm{N}$, and $M$ stages. More importantly, this study believed that the prognostic scoring system should consider treatment strategies and demographic factors, which would improve predictive performance and clinical decisions for individuals.

Surgical resection is the therapeutic mainstay for SIA presenting as a locoregional disease. The nomograms displayed that missed surgery was the worst prognostic factor, even worse than metastatic disease. The pancreaticoduodenectomy with negative margins and an adequate lymph-node evaluation should be performed for the first and second portions of the duodenum. Wide local excision and regional lymph-node dissection are indicated 
for the third and fourth portions of the duodenum and Jejunum or ileal adenocarcinomas. The distal or terminal ileum should be treated by right colectomy. Moreover, the number of regional lymph nodes to be evaluated should be determined. Using the SEER database, two recent researches distinguished either $\geq 8$ or $\geq 10$ lymph nodes as the optimal number $(13,14)$. This study's findings are inconsistent with previous research since survival benefits were significantly greater for patients with more than $12 \mathrm{RNE}$, referring to colorectal cancer, compared to 8-11 RNE.

The number of patients with adjuvant chemotherapy increased from $8.1 \%$ in 1985 to $22.2 \%$ in 2005 in the National Cancer Database $(4,15)$. In this study, $43.70 \%$ of SIA patients, a relatively low percentage, received chemotherapy in this study, which included data from 20042016. Adjuvant chemotherapy was expected to be beneficial despite the lack of randomized trials. A retrospective study including 54 patients revealed that adjuvant therapy was associated with improvement of DFS (HR 0.27; 95\% CI, $0.07-0.98, \mathrm{P}=0.05)$ in multivariate analysis (16). Czaykowski revealed that patients with chemotherapy had 15.6-month OS, while those without chemotherapy only had 7.7-month OS in the data from the registry of British Columbia (17). Moreover, a previous study showed an obvious increase in overall survival in the chemotherapy cohort (12 vs. 2 months, $\mathrm{P}=0.02$ ) (9). Similarly, the prognosis in this study was significantly better for patients in the chemotherapy group compared to the non-chemotherapy group. The intuitive nomograms can also be used to encourage patients with small bowel cancer to receive treatment actively.

A small sample study reported that radiotherapy demonstrated a trend towards improved 5-year overall survival (18). However, this study with data from the SEER database cannot support this tendency. Clinicians need to re-evaluate the value of radiotherapy, as radiation may injure the small intestine and surrounding tissues. Besides, the difference between MCC/SRCC, being considered highly malignant, and adenocarcinomas was non-significant. In addition, age, marital status, and pathological grade were also related to the survival of small bowel cancer, which was consistent with colorectal cancer (19-22).

The advantages of the nomograms are (I) superior survival prediction ability to the AJCC stage, (II) ability to determine the value of treatment strategies, and (III) ability to distinguish more than $12 \mathrm{RNE}$ as the optimal number. This study had some limitations. First, as a retrospective study, the nomograms still need to be validated by prospective studies in the future. Second, this study did not include some important factors, such as CEA and CA-199, among others, which were missing in the SEER database. However, the excellent sensitivity, specificity, and outstanding clinical value of the nomograms for SIA are the strengths of this study.

\section{Conclusions}

This study built and verified nomograms to predict OS and CSS for rare SIA, showing that they may serve as an excellent tool to augment the clinically available evidence to facilitate the discussion between SIA patients and clinicians regarding therapeutic choice.

\section{Acknowledgments}

The authors acknowledge the efforts of the Surveillance, Epidemiology, and End Results (SEER) Program tumor registries in the creation of the SEER database. The interpretation and reporting of these data are the sole responsibility of the authors.

Funding: This study was supported by the Nature Scientific Foundation of China (Grant No. 81702956); the StrategyOriented Special Project of Central South University in China (Grant No. ZLXD2017003); the Natural Science Foundation of Hunan Province (Grant No. 2020JJ4903 and 2020JJ5920); and The Colorectal cancer medical seed research fund project named "Effect and mechanism of YAP1 on EGFR resistance in K-ras wild-type metastatic colorectal cancer" from the Beijing Bethune Public Welfare Foundation.

\section{Footnote}

Reporting Checklist: The authors have completed the TRIPOD reporting checklist. Available at https://dx.doi. org/10.21037/apm-21-600

Conflicts of Interest: All authors have completed the ICMJE uniform disclosure form (available at https://dx.doi. org/10.21037/apm-21-600). Dr. FT reports receiving the following funding: The Nature Scientific Foundation of China (Grant No.81702956), The Strategy-Oriented Special Project of Central South University in China (Grant No. ZLXD2017003), The Natural Science Foundation of Hunan Province (Grant No.2020JJ4903 and 2020JJ5920) and The Colorectal cancer medical seed research fund project named "Effect and mechanism of YAP1 on EGFR 
resistance in K-ras wild-type metastatic colorectal cancer" from the Beijing Bethune Public Welfare Foundation. Dr. DW reports receiving funding from China Scholarship Council. The other authors have no conflicts of interest to declare.

Ethical Statement: The authors are accountable for all aspects of the work in ensuring that questions related to the accuracy or integrity of any part of the work are appropriately investigated and resolved. The study was conducted in accordance with the Declaration of Helsinki (as revised in 2013).

Open Access Statement: This is an Open Access article distributed in accordance with the Creative Commons Attribution-NonCommercial-NoDerivs 4.0 International License (CC BY-NC-ND 4.0), which permits the noncommercial replication and distribution of the article with the strict proviso that no changes or edits are made and the original work is properly cited (including links to both the formal publication through the relevant DOI and the license). See: https://creativecommons.org/licenses/by-nc-nd/4.0/.

\section{References}

1. Raghav K, Overman MJ. Small bowel adenocarcinomas-existing evidence and evolving paradigms. Nat Rev Clin Oncol 2013;10:534-44.

2. Aparicio T, Zaanan A, Svrcek M, et al. Small bowel adenocarcinoma: epidemiology, risk factors, diagnosis and treatment. Dig Liver Dis 2014;46:97-104.

3. Haselkorn T, Whittemore AS, Lilienfeld DE. Incidence of small bowel cancer in the United States and worldwide: geographic, temporal, and racial differences. Cancer Causes Control 2005;16:781-7.

4. Bilimoria KY, Bentrem DJ, Wayne JD, et al. Small bowel cancer in the United States: changes in epidemiology, treatment, and survival over the last 20 years. Ann Surg 2009;249:63-71.

5. Sellner F. Investigations on the significance of the adenoma-carcinoma sequence in the small bowel. Cancer 1990;66:702-15.

6. Vogelstein B, Fearon ER, Hamilton SR, et al. Genetic alterations during colorectal-tumor development. N Engl J Med 1988;319:525-32.

7. Calman KC. Why are small bowel tumours rare? An experimental model. Gut 1974;15:552-4.

8. Halfdanarson TR, McWilliams RR, Donohue JH, et al. A single-institution experience with 491 cases of small bowel adenocarcinoma. Am J Surg 2010;199:797-803.

9. Dabaja BS, Suki D, Pro B, et al. Adenocarcinoma of the small bowel: presentation, prognostic factors, and outcome of 217 patients. Cancer 2004;101:518-26.

10. Overman MJ, Hu CY, Kopetz S, et al. A population-based comparison of adenocarcinoma of the large and small intestine: insights into a rare disease. Ann Surg Oncol 2012;19:1439-45.

11. Legué LM, Bernards N, Gerritse SL, et al. Trends in incidence, treatment and survival of small bowel adenocarcinomas between 1999 and 2013: a populationbased study in The Netherlands. Acta Oncol 2016;55:1183-9.

12. Howe JR, Karnell LH, Menck HR, et al. The American College of Surgeons Commission on Cancer and the American Cancer Society. Adenocarcinoma of the small bowel: review of the National Cancer Data Base, 19851995. Cancer 1999;86:2693-706.

13. Nicholl MB, Ahuja V, Conway WC, et al. Small bowel adenocarcinoma: understaged and undertreated? Ann Surg Oncol 2010;17:2728-32.

14. Overman MJ, Hu CY, Wolff RA, et al. Prognostic value of lymph node evaluation in small bowel adenocarcinoma: analysis of the surveillance, epidemiology, and end results database. Cancer 2010;116:5374-82.

15. Lepage C, Bouvier AM, Manfredi S, et al. Incidence and management of primary malignant small bowel cancers: a well-defined French population study. Am J Gastroenterol 2006;101:2826-32.

16. Overman MJ, Kopetz S, Lin E, et al. Is there a role for adjuvant therapy in resected adenocarcinoma of the small intestine. Acta Oncol 2010;49:474-9.

17. Czaykowski P, Hui D. Chemotherapy in small bowel adenocarcinoma: 10-year experience of the British Columbia Cancer Agency. Clin Oncol (R Coll Radiol) 2007;19:143-9.

18. Kelsey CR, Nelson JW, Willett CG, et al. Duodenal adenocarcinoma: patterns of failure after resection and the role of chemoradiotherapy. Int J Radiat Oncol Biol Phys 2007;69:1436-41.

19. Li Y, Zhao L, Güngör C, et al. The main contributor to the upswing of survival in locally advanced colorectal cancer: an analysis of the SEER database. Therap Adv Gastroenterol 2019;12:1756284819862154.

20. Wang Z, Wang Y, Yang Y, et al. A competing-risk nomogram to predict cause-specific death in elderly patients with colorectal cancer after surgery (especially for 
colon cancer). World J Surg Oncol 2020;18:30.

21. Zheng P, Lai C, Yang W, et al. Nomogram predicting cancer-specific survival in elderly patients with stages I-III colon cancer. Scand J Gastroenterol
2020;5 5:202-8.

22. Li C, Pei Q, Zhu H, et al. Survival nomograms for stage III colorectal cancer. Medicine (Baltimore) 2018;97:e13239.

Cite this article as: Wang D, Li C, Li Y, Liu W, Zhao L, Güngör C, Tan F, Zhou Y. Specific survival nomograms based on SEER database for small intestine adenocarcinoma. Ann Palliat Med 2021;10(7):7440-7457. doi: 10.21037/apm-21-600 